\title{
Thermionic-enhanced near-field thermophotovoltaics
}

\author{
A. Datas , R. Vaillon
}

Keywords:

Nanoscale energy conversion

Thermophotovoltaics

Thermionics

Near-field radiation

\begin{abstract}
Solid-state heat-to-electrical power converters are thermodynamic engines that use fundamental particles, such as electrons or photons, as working fluids. Virtually all commercially available devices are thermoelectric generators, in which electrons flow through a solid driven by a temperature difference. Thermophotovoltaics and thermionics are highly efficient alternatives relying on the direct emission of photons and electrons. However, the low energy flux carried by the emitted particles significantly limits their generated electrical power density potential. Creating nanoscale vacuum gaps between the emitter and the receiver in thermionic and thermophotovoltaic devices enables a significant enhancement of the electron and photon energy fluxes, respectively, which in turn results in an increase of the generated electrical power density. Here we propose a thermionicenhanced near-field thermophotovoltaic device that exploits the simultaneous emission of photons and electrons through nanoscale vacuum gaps. We present the theoretical analysis of a device in which photons and electrons travel from a hot $\mathrm{LaB}_{6}$-coated tungsten emitter to a closely spaced $\mathrm{BaF}_{2}$-coated InGaAs photovoltaic cell. Photon tunnelling and space charge removal across the nanoscale vacuum gap produce a drastic increase in flux of electrons and photons, and subsequently, of the generated electrical power density. We show that conversion efficiencies and electrical power densities of $\sim 30 \%$ and $\sim 70 \mathrm{~W} / \mathrm{cm}^{2}$ are achievable at $2000 \mathrm{~K}$ for a practicable gap distance of $100 \mathrm{~nm}$, and thus greatly enhance the performances of stand-alone near-field thermophotovoltaic devices $\left(\sim 10 \%\right.$ and $\left.\sim 10 \mathrm{~W} / \mathrm{cm}^{2}\right)$. A key practical advantage of this nanoscale energy conversion device is the use of grid-less cell designs, eliminating the issue of series resistance and shadowing losses, which are unavoidable in conventional near-field thermophotovoltaic devices.
\end{abstract}

\section{Introduction}

Conversion of heat into electricity is the backbone of all modern economies, generating most of world's electric power. This includes non-renewable (gas, coal, nuclear) and renewable (solar thermal) power plants. Virtually all heat engines in operation today are dynamic systems, involving generation of mechanical energy, typically a fluid flow, as an intermediate step for conversion of heat into electricity. Thermoelectric generators (TEG) [1] are a solid state alternative to dynamic systems, but they are fundamentally limited by conduction heat losses, which preclude the achievement of large temperature gradients and conversion efficiencies, which are typically below $10 \%$ $[2,3]$.

The scarch of a highly efficient alternative to TEG has been the focus of a continuous research effort since early 1950's. The two main alternatives rely on the direct emission of photons (thermophotovoltaics)
[4,5] or electrons (thermionics) $[6,7,8]$. In thermionics (TIC), electrons thermally emitted from a hot cathode are collected in a cold anode (or collector), and thus produce an electrical current. In thermophotovoltaics (TPV), thermally radiated photons are absorbed in a lowbandgap semiconductor and excite electron-hole pairs, which are selectively collected to produce an electrical current. Both concepts use a noncontact approach, in which the solid continuity between the hot and cold reservoirs is broken. This fully eliminates the phonon transport or heat conduction losses, which are unavoidable in TEG, and theoretically enables much higher conversion efficiencies. Conversely, the energy flux and generated power density is significantly lower due to the lower energy flux carried by radiated photons and electrons. For instance, the highest conversion efficiency of TPV devices reported so far is $24 \%$, but the power density $\left(0.79 \mathrm{~W} / \mathrm{cm}^{2}\right.$ at $1312 \mathrm{~K}$ [9]) is much lower than that of current state of the art TEG (today's record is $22 \mathrm{~W} /$ $\mathrm{cm}^{2}$ at $868 \mathrm{~K} \mathrm{[10]).} \mathrm{Much} \mathrm{higher} \mathrm{power} \mathrm{densities,} \mathrm{in} \mathrm{the} \mathrm{range} \mathrm{of}$ 
$17-25 \mathrm{~W} / \mathrm{cm}^{2}$, were experimentally demonstrated for TIC operating at $1400-1700{ }^{\circ} \mathrm{C}$, but with significantly lower conversion efficiencies in the range of $7-11 \%[8,11]$.

Increasing power density and conversion efficiency of TPV and TIC is an important and active field of research today. Concerning TIC, most of the research focus on finding materials having a low workfunction, and device architectures that eliminate space charge (e.g. micro-spacing the cathode and collector) $[6,7,8]$. For TPV, the main alternatives consist of developing high quality TPV cells with extended spectral response [12], or exploring novel device concepts that enable increasing the energy flux of radiative power. This can be done by using light-pipes [13] or near-field arrangements [14], the latter one having the greatest theoretical potential. Near-field TPV (nTPV) was proposed by Pan et al. [14] in 2000 as a variation of TPV in which the emitter and the photovoltaic (PV) cell are located at nanometric distances, so that photons can tunnel through the nanoscale gap and produce a significant enhancement of radiative energy transfer and generated power density. This concept has been thoroughly assessed from the theoretical point of view during the last two decades $[15,16,17]$. Very recently, the concept has been proven experimentally by measuring a 40 -fold enhancement of the TPV output power at a gap distances of less than $100 \mathrm{~nm}$ [18].

In this article we establish a thermionic-enhanced near-field thermophotovoltaic (nTiPV) device, in which both the emitter and the TPV cell are covered with low workfunction coatings (enabling the emission and collection of electrons) and separated by nanoscale vacuum gaps. The nanometric spacing provides both space-charge removal and nearfield radiative enhancement, and produces a drastic increase of the flow of electrons and photons through the vacuum gap. We will show that this strategy results in a larger power density and conversion efficiency than that of conventional nTPV. Besides, we will explain why nTiPV could represent a more practical and scalable solution than nTPV to realize a highly efficient alternative to TEG.

\section{Theory}

The operational mechanism of nTiPV relies on the recently proposed concept of hybrid thermionic-photovoltaics [19], according to which photons and electrons are thermally emitted from a hot cathode/ emitter towards a closely spaced TPV cell (Fig. 1). Reducing the separation distance between the emitter and the cell to nanometric scales has two consequences: first, it produces a significant enhancement of the radiative power density, and second, it eliminates the space charge effect $[6,7,8]$. The latter is reflected in a reduction of the energy barriers $\phi_{E M}$ and $\phi_{C M}$ (Fig. 1-b) that are opposing electrons' emission, which subsequently produces a drastic enhancement of the electron flux through the nanoscale gap. The emitted electrons are collected at the TPV cell surface (named collector), where they recombine with the holes that are generated within the TPV cell upon photon absorption. The photogenerated electrons are extracted from the rear-side of the TPV cell and re-injected to the emitter thorough the external wiring, creating an external current $(J)$. A non-negligible lead resistance $\left(R_{\text {lcad }}\right)$ must be considered and carefully optimized to fulfil an existing tradeoff between electrical and thermal conductivity.

In this arrangement, the output voltage is enhanced with respect to the stand-alone TPV (TIC) due to the additional voltage generated by the TIC (TPV) stage, i.e. $V_{T I}\left(V_{P V}\right)$, as illustrated in the band-diagram of Fig. 1-b. On the other hand, the flux of thermionically emitted electrons must equal the external TPV's photogenerated current. Thus, if the thermionic current is lower than the photogenerated one, the TPV cell will be biased near open-circuit. The reverse condition is also true: if the thermionic current is higher than that of photogenerated one in the TPV cell, the thermionic sub-device will be biased at higher voltages than that of the maximum power point (MPP) to match the current photogenerated in the TPV cell. Therefore, in order to fully exploit the contribution of both sub-devices, it is desirable that both thermionic and photovoltaic currents coincide at their respective MPP conditions.
However, as we will see in the discussion (section 4), this condition is not strictly necessary to produce a noticeable enhancement of power density and efficiency with respect to nTPV.

It is worth mentioning that near-field operation is unacceptable for conventional TIC, where radiative losses must be minimized. Thus, an optimal gap distance exists for conventional TIC that fulfils a trade-off between electron and photon energy fluxes [20]. On the contrary, nearfield radiative enhancement is beneficial for nTiPV, where evanescent waves are effectively converted into electricity in the TPV cell.

Apart from the higher energy flux, we show that a key advantage of nTiPV is that the entire front TPV cell surface behaves as a transparent electrode, enabling a 1-D carrier transport with no lateral (2-D) current flow within the TPV cell. This avoids the use of front metal grids, typically needed in nTPV devices, and virtually eliminates the ohmic and grid-shadowing losses. The absence of front metal grids in the nTiPV design significantly simplifies the practical implementation of nanoscale gaps between the emitter and the cell, then eventually enabling the scalability of this technology.

The far-field counterpart of this device [19] is being experimentally implemented in ultra-high vacuum (UHV) conditions by using dielectric micro-spacers between the emitter and the TPV cell, along with a combination of borides and fluorides as thermionic coatings [21,22]. Thermally and electrically insulated micro-spacers that withstand large temperature gradients have already enabled the experimental demonstration of micron-gap TIC [23,24]. Sub-micron separation distances have been also experimentally realized in the frame of near-field thermal radiation experimentations $[25,26,27,28]$. Current research efforts target the use of such nano-spacers into nTPV devices [29]. The proposed conceptual device will eventually take advantage of all these developments, which are directly transferrable to the experimental implementation of nTiPV devices.

\section{Methods}

In this article, we examine one possible implementation of nTiPV (Fig. 1) in which the emitter is made of tungsten coated with a thin $(10 \mathrm{~nm}) \mathrm{LaB}_{6}$ layer, which is regarded as an ideal thermionic cathode due to its low workfunction (2.5-3 eV) and high electrical conductivity $[7,8,22]$. The collector consists of an extremely thin $(1-3 \mathrm{~nm}) \mathrm{BaF}_{2}$ layer, which can be directly deposited on the TPV cell front surface to provide a very low workfunction, in the range of $1-2 \mathrm{eV}$ [22], and negligible absorptance. The TPV cell consists of a thin InGaAs p-n junction $(0.4 \mu \mathrm{m}$ p-region on top of a $0.7 \mu \mathrm{m}$ n-region $)$, whose low bandgap $(0.74 \mathrm{eV})$ enables photo-generation of electron-hole pairs from absorption of thermal radiation emitted at temperatures comprised between 1400 and $2000 \mathrm{~K}$ (see section 1 of the Supplementary Information). The rear gold reflector turns back to the emitter the photons not absorbed in the cell, and thus contribute to boosting conversion efficiency. This specific architecture assumes that the collector layer does not modify the TPV cell band diagram in a way that holes could not diffuse towards the collector. This is a reasonable assumption provided that the high doping of the $\mathrm{p}$-InGaAs layer $\left(8 \cdot 10^{17} \mathrm{~cm}^{-3}\right)$ will enable a high tunnelling probability (field emission) if a barrier is formed at the $\mathrm{p}-\mathrm{InGaAs} / \mathrm{BaF}_{2}$ interface. More details on the material selection criteria are given in section 1 of the Supplementary Information.

Analysis of the nTiPV device described above requires the calculation of the total net flux of photons and electrons through the vacuum gap separating the emitter and the TPV cell. The electron flux between two infinite parallel-plane surfaces can be described by the Langmuir theory [6], which assumes one-dimensional and collision-less electron flow. For the photons, the energy flux is calculated using fluctuational electrodynamics [30] and the S-matrix method for 1D-layered media [31].

According to Langmuir theory, the flow of electrons is determined by the electrostatic potential $\psi$ in the inter-electrode gap. This potential 


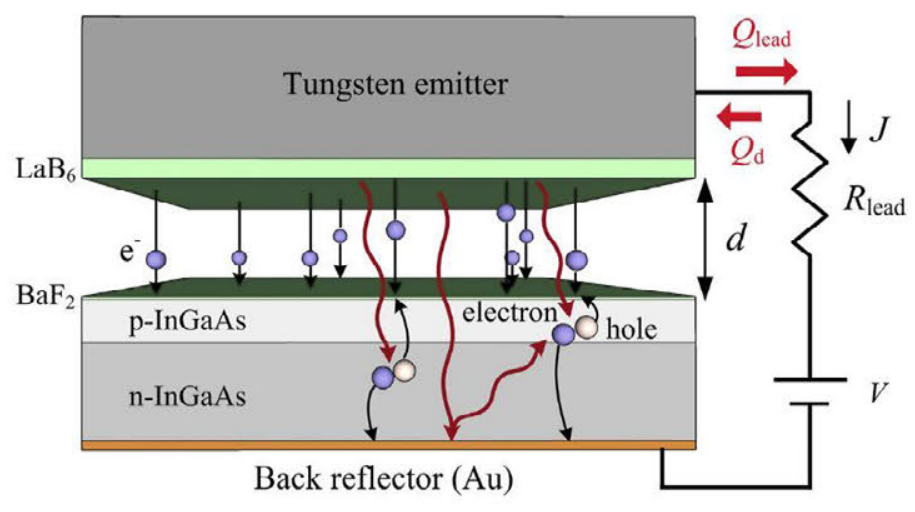

(a)

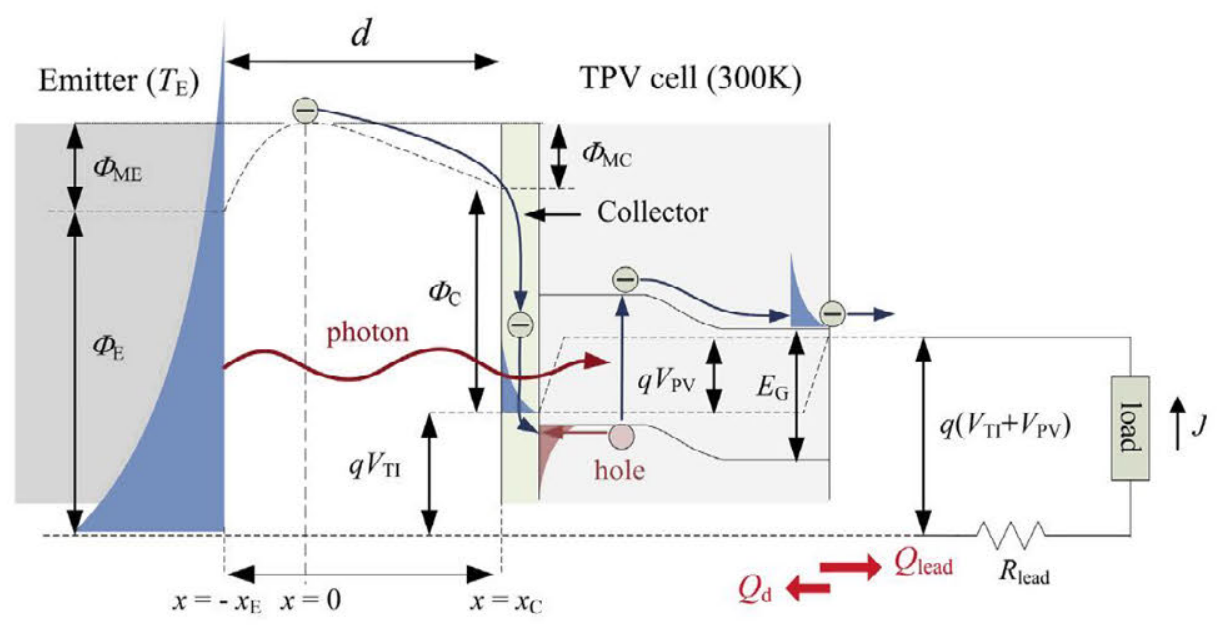

(b)

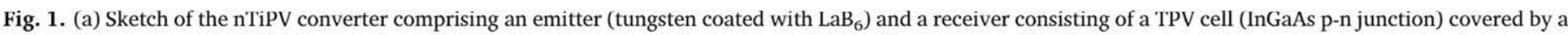

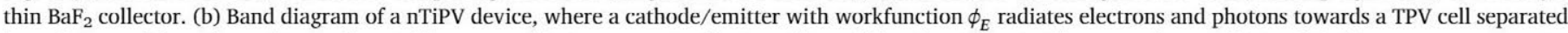

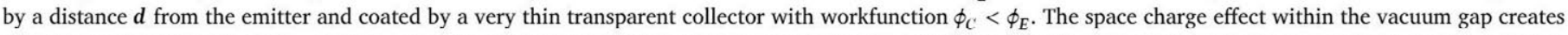

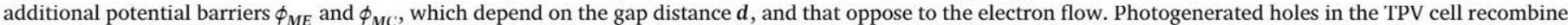

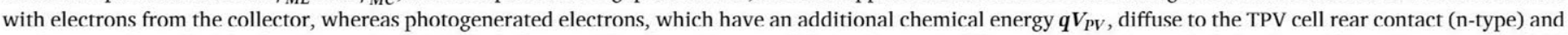

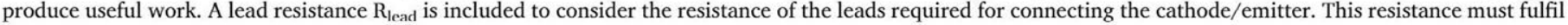
a trade-off between thermal and electrical conductivity.

is obtained by solving Poisson's equation $d^{2} \psi / d x^{2}=-q n_{e}(x) / \varepsilon_{0}$ together with the Vlasov-Poisson approximation for the electron distribution function $f_{e}\left(x, v_{e}\right)$, which is used to determine the electron density $n_{e}(x)=\int f_{e}\left(x, v_{e}\right) d v_{e}$ at a position $x$ within the gap, in the nonrelativistic zero-magnetic field limit. Poisson's equation can be solved analytically by assuming a half-Maxwellian distribution for the velocities $v_{e}$ of thermionically emitted electrons at $x=0 f_{e}\left(x=0, v_{e}\right)$, where the electrostatic potential is maximum and therefore, there are no accelerating or decelerating fields (Fig. 1-b). Following the previous procedure, the relation between electrostatic potential and position within the gap $(x)$ can be derived [6], leading to:

$\xi=\mp \int_{0}^{\gamma} \frac{d t}{\left[e^{t}-1 \pm e^{t} \operatorname{erf}(\sqrt{t}) \mp 2(t / \pi)^{1 / 2}\right]^{1 / 2}}$ for $\xi \lessgtr 0$

where $\xi=\frac{x}{x_{0}}$ is the dimensionless distance, $x_{o}=\left(\frac{\varepsilon_{0}^{2} k^{3}}{2 \pi m_{e} q^{2}}\right)^{1 / 4} \frac{T_{L}^{3 / 4}}{I_{T I}^{1 / 2}}$ is the normalization distance, $J_{T I}$ is the thermionic current density, $\varepsilon_{0}$ is the vacuum permittivity, $m_{e}$ and $q$ are the electron mass and charge, $k$ is the Boltzmann constant, and $\gamma=\frac{q \phi}{k T_{E}}$ is named "dimensionless motive", with $\phi=q \psi$ being the electron motive. Equation (1) can be used to determine the barriers $\phi_{E M}$ and $\phi_{C M}$ (Fig. 1-b) that will ultimately determine the current-voltage characteristic of the thermionic stage. $\phi_{E M}$ can be obtained by combining equation (1) with the Richardson equation, which determines the current density $J_{T T}$ in the case of negligible collector back-emission as [6]:

$J_{T I}=A T_{E}^{2} e^{\frac{-q\left(\phi_{E}+\phi_{E M}\right)}{k T_{E}}}=J_{E S} e^{\frac{-q \phi_{E M}}{k T_{E}}}$

where $\phi_{E M}$ is an additional barrier added to that of the emitter workfunction. This equation is valid for current densities higher than the socalled critical current $J_{R}$, which represents the situation at which the electrostatic potential is maximum at the collector surface, i.e. $x_{C}=0$, $x_{E}=-d$ (see Fig. 1-b). At this critical point, the dimensionless distance is given by $\xi=\frac{-d}{x_{0}\left(J_{R}\right)}$, so that it can be expressed as a function of $\phi_{E M}$ by making use of the Richardson equation (2) for $J_{R}$. The resultant expression $\xi\left(\phi_{E M}\right)$ can be introduced into equation (1) by making $\gamma=\frac{q \phi_{E M}}{k T_{E}}$ to finally obtain $\phi_{E M}$ and $J_{R}$.

For current densities greater than $J_{R}\left(J_{E S}>J_{T I}>J_{R}\right)$, a decelerating (accelerating) field exists near the emitter (collector). This situation is 
named space charge mode and it is the one depicted in Fig. 1-b. In this case, $\phi_{E M}$ can be readily calculated from the Richardson equation (2), while the calculation of $\phi_{C M}$ requires determination of the distances $x_{E}$ (between the emitter and the maximum of the electrostatic potential) and $x_{C}$ (between the collector and the maximum motive). $x_{E}$ is obtained by solving equation (1) for $\gamma=\frac{q \phi_{E M}}{k T_{E}}$ and $\xi=\frac{-x_{E}}{x_{0}}$, and the distance $x_{C}$ can be readily obtained as $x_{C}=d-x_{E}$. Then, $\phi_{C M}$ can be calculated by solving equation (1) for $\gamma=\frac{q \phi_{C M}}{k T_{E}}$ and $\xi=\frac{x_{C}}{x_{0}}$. Once both $\phi_{C M}$ and $\phi_{E M}$ are known for every value of $J_{T I}$, the internal thermionic voltage $V_{T I}$ is finally calculated as $q V_{T I}=\phi_{E}+\phi_{E M}-\phi_{C}-\phi_{C M}$ (see Fig. 1-b). In the case of $J_{T I}<J_{R}$, the inter-electrode potential opposes to the electrons' flow at any position in the gap. In this situation (named retarding mode) the thermionic voltage is directly given by $V_{T I}=-\phi_{C}-\frac{k T_{E}}{q} \ln \left(\frac{J_{T I}}{A T_{E}^{2}}\right)[6]$.

Following the previous procedure, the values of $V_{T I}, \phi_{E M}$ and $\phi_{C M}$ can be determined as a function of the current density $J_{T I}$, and the energy flux of electrons can be readily calculated [6]:

$$
Q_{e l}=J \frac{\left(\phi_{\max }+2 k T_{E}\right)}{e}, \text { where } \phi_{\max }=\left\{\begin{array}{lll}
\phi_{E}+\phi_{M E} & \text { for } & J>J_{R} \\
\phi_{C}+V_{T I} & \text { for } & J<J_{R}
\end{array}\right.
$$

It must be noticed that the method described above assumes that electrons are thermionically emitted from the cathode, independently of the gap distance. When spacing between the emitter and the receiver is very small (well below $\sim 10 \mathrm{~nm}$ ), electron tunneling should be also considered. Electron tunneling was proposed for cooling in thermotunneling devices $[32,33,34]$ and could be useful also for power generation in nTiPV converters.

In order to determine the nTiPV conversion efficiency, an energy balance has to be established considering all kinds of energy carriers. The main contribution to the energy balance is the heat carried by the radiated photons and electrons, but also due to heat conduction through the leads (Fig. 1). According to the Wiedemann-Franz law [6], the minimum amount of heat lost through the leads ultimately depends on the lead electrical resistance $R_{\text {lead }}$. Assuming an average lead temperature of $\left(T_{E}+T_{C}\right) / 2$, this minimum heat is given by $Q_{\text {lead }}=L\left(T_{E}^{2}-T_{C}^{2}\right) / 2 R_{\text {lead }}$ [6], where $L=\frac{\pi^{2} k^{2}}{3 e^{2}}$ is the Lorentz number of the metal. Besides, half of the heat generated in the leads by Joule effect is turned back to the emitter, which can be calculated as $Q_{d}=S^{2} J^{2} R_{\text {lead }} / 2$, where $S$ is the device area, equal to $1 \mathrm{~cm}^{2}$ in the current study.

Finally, the efficiency of the nTiPV converter can be calculated by:

$\eta=\frac{\left[S J\left(V_{T I}-S J R_{\text {lead }}+V_{P V}\right)\right]_{\max }}{S\left(Q_{e l}+Q_{p h}\right)+Q_{\text {lead }}-Q_{d}}$

where $\left[S J\left(V_{T I}-S J R_{\text {lead }}+V_{P V}\right)\right]_{\max }$ is the maximum output power (in Watts) of the nTiPV converter, which is obtained at a certain voltage $V=V_{T I}-S J R_{\text {lead }}+V_{P V}$, given that the values of $V_{T I}$ and $V_{P V}$ are constrained by the current-match condition $J=J_{T I}=J_{P V}$. Notice that equation (4) neglects the heat flow from the emitter to the TPV cell through the nano-spacers that will eventually separate the emitter from the TPV cell. These losses are not accounted for in this analysis because they could be minimized by practical means, e.g. by using tapered spacers with very small contact area [28,29], and therefore, they do not represent a fundamental source of losses of this concept.

The remaining variables that must be calculated are the net photon energy flux $\left(Q_{p h}\right)$ and the TPV current-voltage characteristic $J_{P V}-V_{P V}$. The net photon energy flux is calculated using fluctuational electrodynamics [30] and the S-matrix method for 1D-layered media [31]. The multilayer system (Fig. 1) is composed of 4 layers sandwiched between two semi-infinite media which are respectively made of tungsten (thermal emitter, semi-infinite), lanthanum hexaboride ( $\mathrm{LaB}_{6}$ cathode, $10 \mathrm{~nm}$ thick), vacuum gap (variable thickness $d$ ), barium fluoride $\left(\mathrm{BaF}_{2}\right.$ collector, $1 \mathrm{~nm}$ thick), p-doped $\operatorname{In}_{0.53} \mathrm{Ga}_{0.47}$ As $\left(N_{A}=810^{17} \mathrm{~cm}^{-3}\right.$, $0.4 \mu \mathrm{m}$ thick), n-doped $\operatorname{In}_{0.53} \mathrm{Ga}_{0.47} \mathrm{As}\left(N_{D}=210^{17} \mathrm{~cm}^{-3}, 0.7 \mu \mathrm{m}\right.$ thick), gold back reflector (semi-infinite). A discussion on the selection of these particular materials and the associated property data is presented in section 1 of the Supplementary Information. Calculations are made in the angular frequency interval $\left[7.610^{13}-7.710^{15}\right] \mathrm{rad} / \mathrm{s}$ (wavelength interval [0.245-24.785] $\mu \mathrm{m}$, photon energy interval [0.050-5.068] eV). This interval covers more than $95 \%$ of the Planck radiation spectrum for the emitter temperatures (1400-2000 K) considered in the simulations. The complex permittivity of each material is required over the aforementioned spectral range. For $\operatorname{In}_{0.53} \mathrm{Ga}_{0.47} \mathrm{As}$, data from [35] is used for photon energies above $0.4 \mathrm{eV}$. Below that energy, the complex permittivity is calculated using a Drude model for absorption by the free carriers and a Lorentz model for absorption by the phonons with accounting for the two-mode optical phonon behavior of III-V ternary compounds [36] (see section 2 of the Supplementary Information for details). The complex permittivity of tungsten is calculated using curve-fitting of tabulated data provided in [37]. Permittivity of lanthanum hexaboride $\left(\mathrm{LaB}_{6}\right)$ is taken from data reported in [38] for photon energies in the interval [2-15] eV, and in [39] for photon energies below $2 \mathrm{eV}$. For missing data for photon energy below $0.28 \mathrm{eV}$, a linear extrapolation is used. The real part of the complex refractive index of barium fluoride $\left(\mathrm{BaF}_{2}\right)$ is taken from a dispersion relation available in [40] for wavelengths comprised between 0.15 and $15 \mu \mathrm{m}$. According to [41], the extinction coefficient is null in this interval. For wavelengths larger than $15 \mu \mathrm{m}$, tabulated data is used [41]. Bulk properties may be inappropriate for a nanometer sized layer, but in the present case it is not a major concern since $\mathrm{BaF}_{2}$ is transparent for photons energies above the bandgap of $\mathrm{In}_{0.53} \mathrm{Ga}_{0.47}$ As. Finally, for gold (Au) tabulated data from experiments made on evaporated gold and reported in [42] are used for wavelengths comprised between 0.3 and $24.93 \mu \mathrm{m}$. For smaller wavelengths, data are taken from [43].

The S-matrix method allows calculating radiation emitted by the tungsten and lanthanum hexaboride thermally emitting layers and absorbed by any layer, as a function of the vacuum gap thickness (d), and over spectral intervals of interest [31]. In particular, radiation power absorbed by the receiver constituted of the collector, the p-n junction and the back reflector, $\left(Q_{p h}\right)$ is computed for determining the maximum (stand-alone) nTPV conversion efficiency as $\eta_{n T P V}=P_{\max } / Q_{p h}$, where $P_{\max }=\left[V_{P V} \cdot J_{P V}\right]_{\max }$ is the PV electrical power at the maximum power point. In order to determine the values of $J_{P V}$ and $V_{P V}$, the p- and ndoped junction layers are discretized in sub-layers in order to calculate radiation power absorbed above the bandgap energy of $\operatorname{In}_{0.53} \mathrm{Ga}_{0.47} \mathrm{As}$ through interband processes, and to infer the electron-hole pair generation rate profile, needed for solving electrical transport equations. Electrical properties of $\operatorname{In}_{0.53} \mathrm{Ga}_{0.47}$ As are required for solving the minority carrier (electron and hole) diffusion equations in the frame of the low-injection approximation. Mobilities of electrons and holes are found in [44] and are respectively equal to $6000 \mathrm{~cm}^{2} \mathrm{~V}^{-1} \mathrm{~s}^{-1}$ and $200 \mathrm{~cm}^{2} \mathrm{~V}^{-1} \mathrm{~s}^{-1}$ for the selected doping concentrations $\left(N_{D}=2\right.$ $10^{17} \mathrm{~cm}^{-3}, N_{A}=810^{17} \mathrm{~cm}^{-3}$ ). Radiative and Auger recombination coefficients are taken from [44], while the impurity recombination lifetime is derived from a curve fitting expression of experimental data as a function of doping density [45] and the Matthiessen's rule (see details in section 2.1 of the Supplementary Information). With these properties, the diffusion equations are solved as in $[46,47,48]$ in order to derive the $J_{P V}-V_{P V}$ characteristic and associated parameters. In particular, the electrical power output and current density at the maximum power point $\left(P_{\max }, J_{P V, \max }\right)$ are respectively used for inferring the TPV converter efficiency and the TIC converter current matching that of the TPV device.

For comparison purposes, stand-alone nTPV devices are also analyzed in this article, for which ohmic losses due to lateral current flow in the top semiconductor p-type layer must be incorporated. In this case, a lumped series resistance is included that results in a lower output voltage given by $\left.V_{P V}\right|_{R_{S} \neq 0}=\left.V_{P V}\right|_{R_{S}=0}-R_{S} J_{P V}$. The value of $R_{S}$ strongly depends on the size and specific layout of the TPV cell. More details on the calculation of $R_{S}$ are given in section 2 of the 

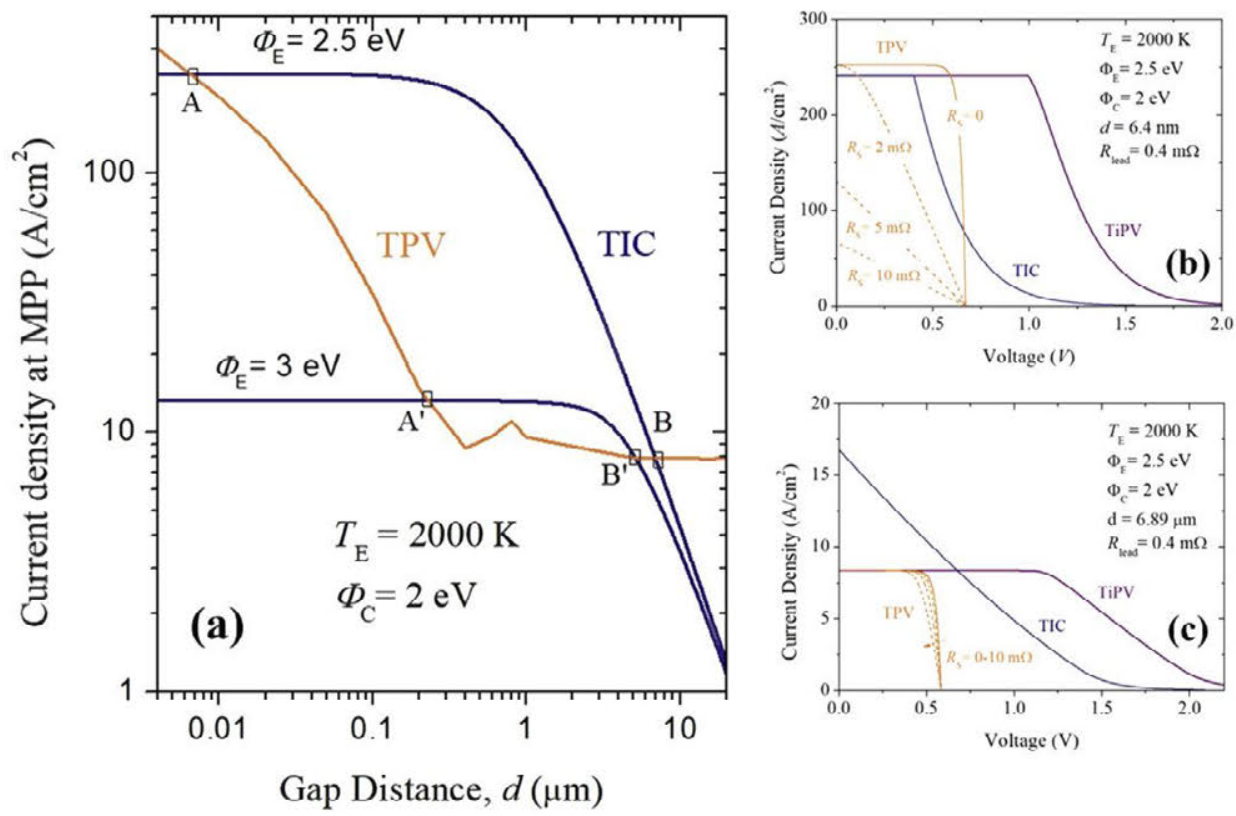

Fig. 2. (a) Current density at the MPP for standalone TPV (with $R_{s}=0$ ) and TIC sub-devices as a function of the gap distance d. (b-c) Current density - Voltage $(\mathrm{J}-\mathrm{V})$ curves for the TiPV devices at current-match condition in the near field (point A) and far field (point B). The J-V curves of independent TPV and TIC sub-devices are superimposed, illustrating the series resistance ef fect of stand-alone TPV cells (dashed J-V curves). The lead resistance $R_{\text {lcad }}$ is optimized independently for the two cases $\left(\phi_{E}=2.5 \mathrm{eV}\right.$ and $\phi_{E}=3 \mathrm{eV}$ ), in order to provide the highest conversion efficiency at the current-match condition in the near field. The device area is $1 \mathrm{~cm}^{2}$.
Supplementary Information.

In order to find optimal parameters leading to the maximum conversion efficiency, the Nelder-Mead algorithm [49] is used. For instance, it is used to search for the MPP voltage of both TPV and TIC converters, as well as the optimum values of $\phi_{E}$ and $R_{\text {lead }}$ that maximize the conversion efficiency under the constraint of current-match at MPP, i.e. $J=\left.J_{P V}\right|_{\max }=\left.J_{T I}\right|_{\max }$.

\section{Results and discussion}

Fig. 2-a shows the current density at MPP for both TIC $\left(J_{T I}\right)$ and TPV $\left(J_{P V}\right)$ independent converters as a function of gap distance for the case with $T_{\mathrm{E}}=2000 \mathrm{~K}$ and two different values of $\phi_{E}$. At distances larger than $\sim 1 \mu \mathrm{m}$, TPV operates in the far-field regime and the TPV photogenerated current density $\left(J_{P V}\right)$ does not depend on distance. At smaller distances, photon tunnelling begins to dominate radiative energy transfer, leading to a drastic rise in photocurrent density. On the contrary, thermionic current is constant for distances below a certain value, at which the space charge is fully eliminated. For larger gap distances, space charge creates an additional barrier $\left(\phi_{E M}\right)$ to electrons' emission that results in a decrease of current density. As a result, there are two crossing points at which both TIC and TPV sub-devices produce the same current at MPP (Fig. 2-a): one in the near field (e.g. points A and $\mathrm{A}^{\prime}$ ) and another in the far field (e.g. points B and B').

As explained in section 2, the series connection between the thermionic and photovoltaic stages in the nTiPV device (Fig. 1) makes desirable that both $J_{T I}$ and $J_{P V}$ coincide at their respective MPP. Thus, points A ( $\left.\mathrm{A}^{\prime}\right)$ and $\mathrm{B}\left(\mathrm{B}^{\prime}\right)$ represent the best-case scenario at which nTiPV fully exploits both TIC and TPV contributions.

The corresponding $J-V$ curves at points A $(d=6.4 \mathrm{~nm})$ and B $(d=6.9 \mu \mathrm{m})$ of Fig. 2-a are shown inFig. 2-b and Fig. 2-c, respectively, along with the $J_{\mathrm{PV}}-V_{\mathrm{PV}}$ and $J_{\mathrm{TI}}-V_{\mathrm{TI}}$ curves of the corresponding standalone TPV and TIC devices. Dashed lines represent the $J_{\mathrm{PV}}-V_{\mathrm{PV}}$ curves assumed to have a lumped series resistance $\left(R_{\mathrm{S}}\right)$ in the range of $2-10 \mathrm{~m}$ $\Omega$ (cell size of $1 \mathrm{~cm}^{2}$ ). Notice that grid-less square TPV cells of $1 \times 1$ $\mathrm{cm}^{2}$ have a lumped resistance in the range of 1-10 $\Omega$ (see section 3 of the Supplementary Information). Reaching such a low range of values for $R_{\mathrm{S}}$ in a stand-alone TPV converter would require relatively dense front-side metallic grids on the TPV cell. Implementing such kind of metallic grids in a near-field arrangement is particularly challenging and would bring additional shadowing and dark-current losses, not accounted for in these simulations. Therefore, the results shown for stand-alone TPV converters in this article must be regarded as the upper bound for their performance. Contrary to that, in nTiPV devices current flows in a single 1-D direction and photogenerated holes are collected from the entire TPV cell front surface. Thus, lateral conduction is fully avoided, resulting in a negligible series resistance and larger power generation capacity, as clearly shown in the $J$-V curves of Fig. 2-b (in the near field).

The negligible ohmic losses, combined with the additional voltage generated in the thermionic stage $\left(V_{\mathrm{TI}}\right)$, produces a noticeable enhancement of the electrical power density of nTiPV. This is illustrated in Fig. 3, which shows the electrical power density as a function of gap distance for both nTPV and nTiPV devices. The figure illustrates the relevance of using low workfunctions for the emitter in the nTiPV device to fully exploit the near-field enhancement at very small gap distances. Large workfunctions produce too low thermionic currents, lower than the photogenerated one, and the TPV cell is subsequently biased near open circuit rather than at the maximum power point. Nevertheless, nTiPV clearly outperforms nTPV for most of the gap distances, despite not fulfilling the current-match condition. Only at

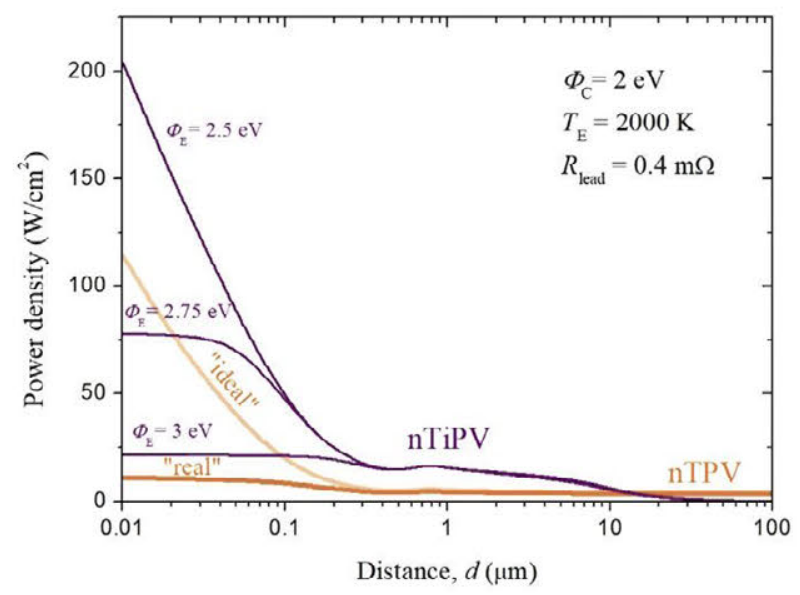

Fig. 3. Electrical power density of nTiPV and nTPV converters as a function of gap distance between the emitter and the TPV cell. Different workfunctions of the emitter $\left(\phi_{\mathrm{E}}\right)$ are considered for nTiPV. "ideal" and "real" nTPV refers to the case with negligible ohmic losses and the more realistic case with a series resistance of $10 \mathrm{~m} \Omega$, respectively. The device area is $1 \mathrm{~cm}^{2}$. 


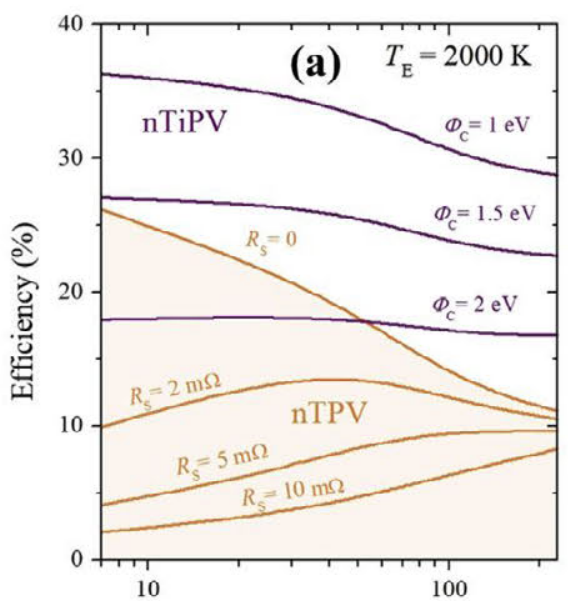

Gap Distance, $d(\mathrm{~nm})$
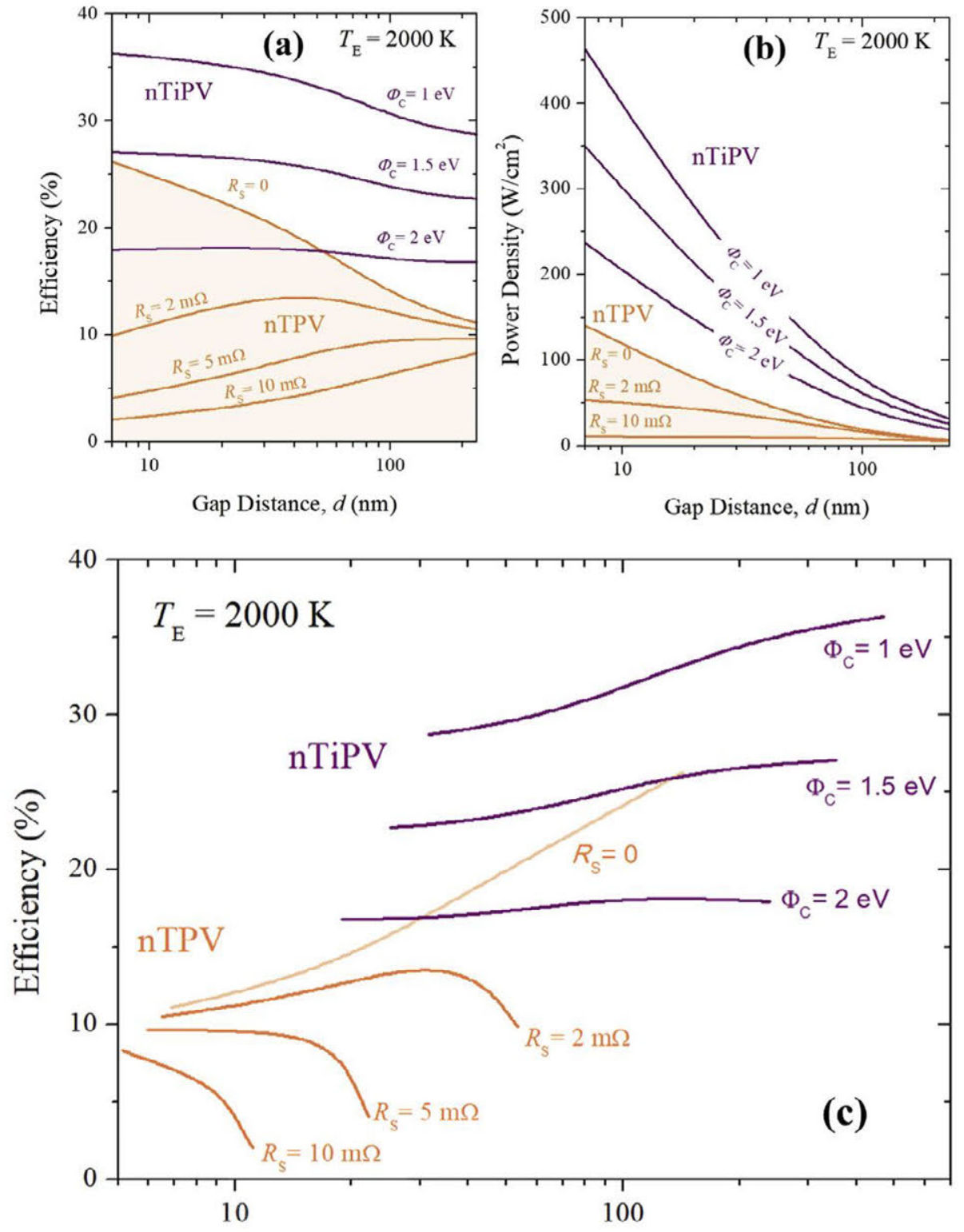

Power Density $\left(\mathrm{W} / \mathrm{cm}^{2}\right)$

Fig. 4. (a) Conversion efficiency and (b) electrical power density of nTiPV and nTPV as a function of gap distance, (c) conversion efficiency as a function of electrical power density for nTiPV and nTPV converters, rearranged from the results shown in (a) and (b). Results are obtained for an emitter temperature of $2000 \mathrm{~K}$. Both the emitter workfunction $\phi_{E}$ and the lead resistance $R_{\text {lead }}$ are optimized. Results for standalone TPV devices with different values of series resistance are superimposed for direct comparison. The device area is $1 \mathrm{~cm}^{2}$.

very large gap distances (above $\sim 10 \mu \mathrm{m}$ ) nTiPV power decreases significantly due to the decreasing thermionic current, which is attributed to the increasing space charge. Fig. 3 shows that the power density of nTiPV can be an order of magnitude greater than that of "real" nTPV devices, assumed to have a series resistance of $10 \mathrm{~m} \Omega \mathrm{cm}^{2}$, and twice as great as the one of "ideal" nTPV, assumed to have no ohmic losses.

Another important observation is that nTiPV enables larger (more practical) gap distances to produce a given electrical power. For instance, a nTiPV device operating at $2000 \mathrm{~K}$ with $\phi_{E}=2.5 \mathrm{eV}, \phi_{C}=2 \mathrm{eV}$ , and $d=100 \mathrm{~nm}$ provides an electrical power density of $\sim 50 \mathrm{~W} / \mathrm{cm}^{2}$ (Fig. 3), while in the best of the cases for "ideal" nTPV, a gap distance of $\sim 40 \mathrm{~nm}$ would be required to provide such a power level. In a more realistic scenario, where ohmic losses are not neglected in the TPV cell, an even shorter gap distance would be required. But most probably, such a high-power density would be unattainable in practice by nTPV at any gap distance.

As mentioned before, the full potential of nTiPV is achieved when both thermionic and photogenerated currents are identical at the respective MPP of both TPV and TIC sub-devices. This case is illustrated in Fig. 4, where conversion efficiency (Fig. 4-a) and electrical power density (Fig. 4-b) of nTiPV and nTPV are shown as a function of gap distance for an optimized emitter workfunction, in the range of $2.5-3 \mathrm{eV}$, and lead resistance, in the range of $0.4-10 \mathrm{~m} \Omega$ (device area of $1 \mathrm{~cm}^{2}$ ), in order to produce the maximum efficiency and the currentmatch between thermionic and photogenerated currents in the near field. It is clearly observed that nTiPV generally outperforms nTPV in terms of both conversion efficiency and electrical power density. This is especially true for low collector workfunctions, resulting in a larger thermionic voltage $V_{\mathrm{TI}}$, and when there are significant ohmic losses in the stand-alone TPV device. For instance, conversion efficiencies and 